\title{
July 2017 Imaging Case of the Month
}

Michael B. Gotway, MD

Department of Radiology

Mayo Clinic Arizona

Scottsdale, Arizona USA

Clinical History: A 56-year-old man with no significant past medical history presented with complaints of cough, shortness of breath, and productive sputum. Frontal and lateral chest radiography (Figure 1) was performed.

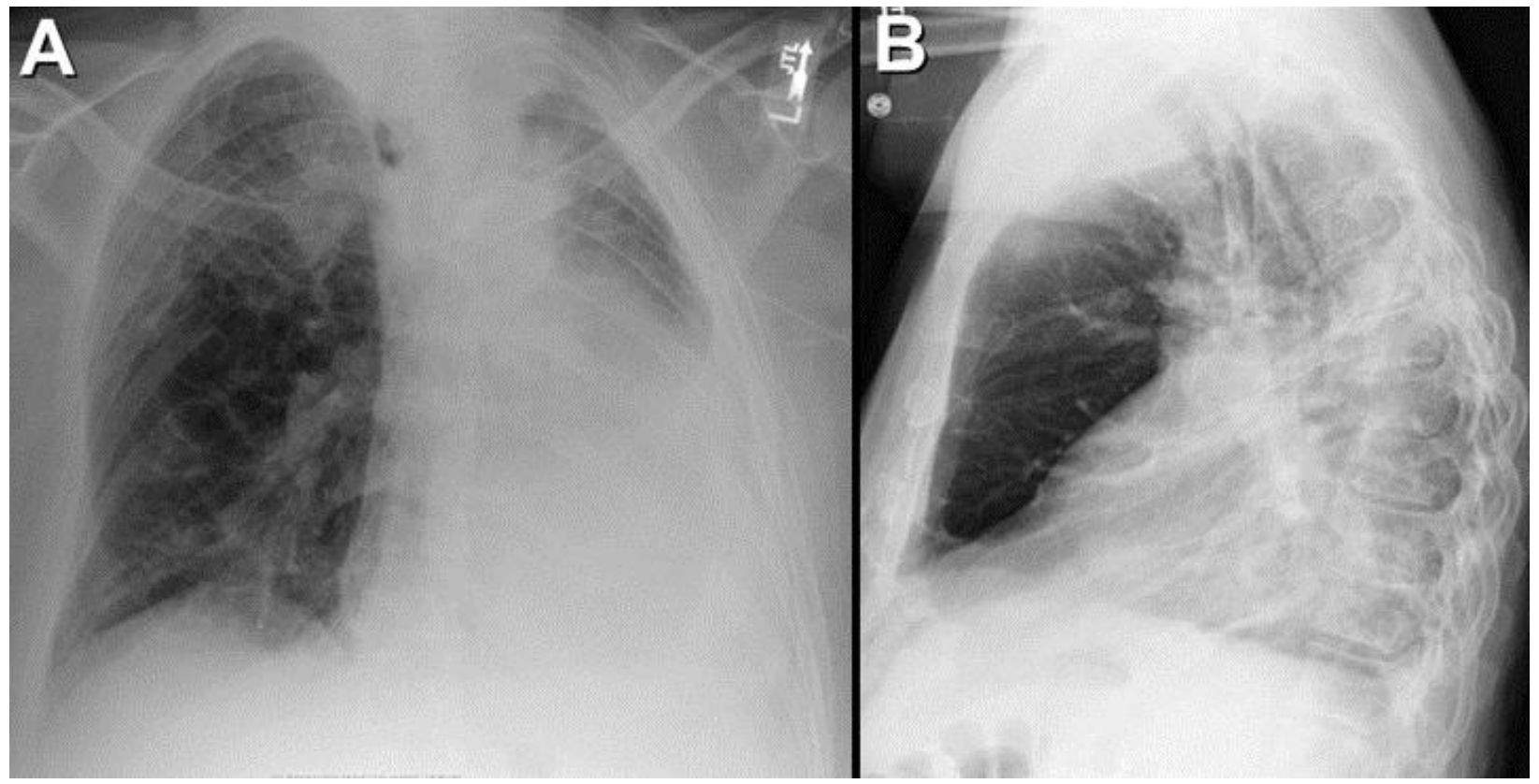

Figure 1. Frontal (A) and lateral $(B)$ chest radiography.

Which of the following statements regarding the chest radiograph is most accurate?

1. The chest radiograph shows a diffuse linear, interstitial pattern

2. The chest radiograph shows a large pleural effusion

3. The chest radiograph shows a mediastinal mass

4. The chest radiograph shows numerous small nodules

5. The chest radiograph shows right lower lobe consolidation 


\section{Correct! \\ 5. The chest radiograph shows right lower lobe consolidation}

The frontal chest radiograph shows patchy right lower lobe consolidation, evidenced by opacity that obscured the right diaphragm. No large pleural effusion is present; the right costophrenic angle appears clear, and the left costophrenic angle cannot be visualized. No nodules are seen and no evidence of a mediastinal mass is present. No linear opacities suggestive of interstitial lung disease is seen.

Which of the following statements regarding other findings on the chest radiograph is most accurate?

1. The chest radiograph also shows an anterior mediastinal mass

2. The chest radiograph also shows extensive left thoracic volume loss

3. The chest radiograph also shows left lower lobe bronchopneumonia

4. The chest radiograph shows a right aortic arch

5. The chest radiograph shows an enlarged right pulmonary artery 


\section{Correct!}

\section{The chest radiograph also shows extensive left thoracic volume loss}

The frontal chest radiograph shows extensive left thoracic volume loss, evidenced by a small left thorax in general and marked shift of the cardiomediastinal structures to the left. No clear left lower lobe bronchopneumonia is seen, although the entire left thoracic base is opacified. No left diaphragm is seen on the lateral projection. The aortic arch is located in its normal, left-sided position. The visible portions of the right pulmonary artery appear normal in size. While the left superior mediastinum appears somewhat prominent on the frontal projection, the retrosternal space on the lateral projection is clear- no anterior mediastinal mass is seen.

Which of the following represent appropriate differential diagnostic considerations for the chest radiographic pattern present?

1. Left lung agenesis

2. Left mainstem bronchial obstruction

3. Post-obstructive air trapping in the right lung

4. Previous left pneumonectomy

5. All of the above 


\section{Correct! \\ 5. All of the above}

All of the listed considerations could result in extensive volume loss affecting one side of the thorax. In this case, a previous left pneumonectomy is certainly expected to produce extensive left thoracic volume loss; left mainstem bronchial obstruction could do the same. Absence of the left lung (agenesis, aplasia) would also produce these findings. Right-sided space occupying lesions, such as pneumothorax, pleural effusion, and postobstructive air trapping can produce contralateral cardiomediastinal shift and thus produce findings similar to those seen on the chest radiograph displayed in Figure

Antimicrobial therapy was initiated for the patient's right lower lobe bronchopneumonia.

Which of the following represents the most appropriate next step for the management of this patient?

1. Assess for left-sided thoracotomy scar

2. Bronchoscopy to assess for bronchial obstruction

3. Repeat chest radiography

4. Thoracic CT

5. Thoracic MRA 


\section{Correct! \\ 1. Assess for left-sided thoracotomy scar}

The simplest and easiest approach to exclude one of the main causes for the appearance of extensive left-sided thoracic volume loss previously listed is to ascertain whether or not the patient has undergone left thoracotomy. This could be accomplished through the performance of a thorough and careful history, but occasionally patients do not provide accurate historical data, even for procedures as major, and presumably as memorable, as a thoracotomy. However, a thoracotomy would leave a visible left chest scar; absence of such is good evidence that previous surgical resection is not the etiology for the patient's left-sided volume loss. Thoracic CT and thoracic MRA could both assess for the various etiologies that may be responsible for the extensive left lung volume loss, but both are premature at this point- if the patient has a thoracotomy scar on the left, then he has undergone previous left pneumonectomy and is simply currently seeking medical attention for a right lower lobe bronchopneumonia, and advanced cross sectional imaging is not needed. Similarly, bronchoscopy is not yet required at this point, and probably would not be indicated unless cross sectional imaging were obtained and did not provide information that clarifies the patient's situation. Repeat chest radiography is unlikely to be beneficial because the chest radiographic findings are very unlikely to be artefactual.

The patient was questioned and examined and he denied any previous surgeries, not was a thoracotomy scar seen.

Which of the following now represents the next most appropriate step for the evaluation of this patient?

1. ${ }^{133}$ Xe-Ventilation - ${ }^{99 m}$ Tc-perfusion scintigraphy

2. ${ }^{18}$ FDG-PET scan

3. ${ }^{68} \mathrm{Ga}$-citrate scan

4. Flexible fiberoptic bronchoscopy

5. Thoracic CT 


\section{Correct! \\ 5. Thoracic CT}

Bronchoscopy may play a role in the evaluation of this patient, given that bronchial obstruction remains as a potential etiology for the patient's now unexplained extensive left-sided thoracic volume loss, but this procedure is premature at this point. Thoracic CT would be capable of non-invasively assessing for right-sided thoracic spaceoccupying lesions that may produce contralateral mediational shift as well as the various potential etiologies capable of producing extensive left-sided volume loss, such as left mainstem endobronchial obstruction, left-sided visceral pleural restriction, and left lung agenesis / aplasia. ${ }^{133}$ Xe-Ventilation - ${ }^{99 m}$ Tc-perfusion scintigraphy could provide some information regarding abnormal ventilation and differential pulmonary perfusion, but it is unlikely that this procedure would allow a confident determination of the cause of the extensive left-sided thoracic volume loss. ${ }^{168}$ Ga-citrate scanning is now seldom used, only occasionally playing a role for the assessment of diffuse lung disease, which is not relevant to this patient. ${ }^{18} \mathrm{FDG}-\mathrm{PET}$ scanning is primarily employed for the assessment and staging of primary intrathoracic malignancy and metastatic disease as well as the evaluation of an indeterminate solitary pulmonary nodule, but would not be of value for this patient at this point in his evaluation.

Unenhanced thoracic CT (Figures 2 and 3) was performed.

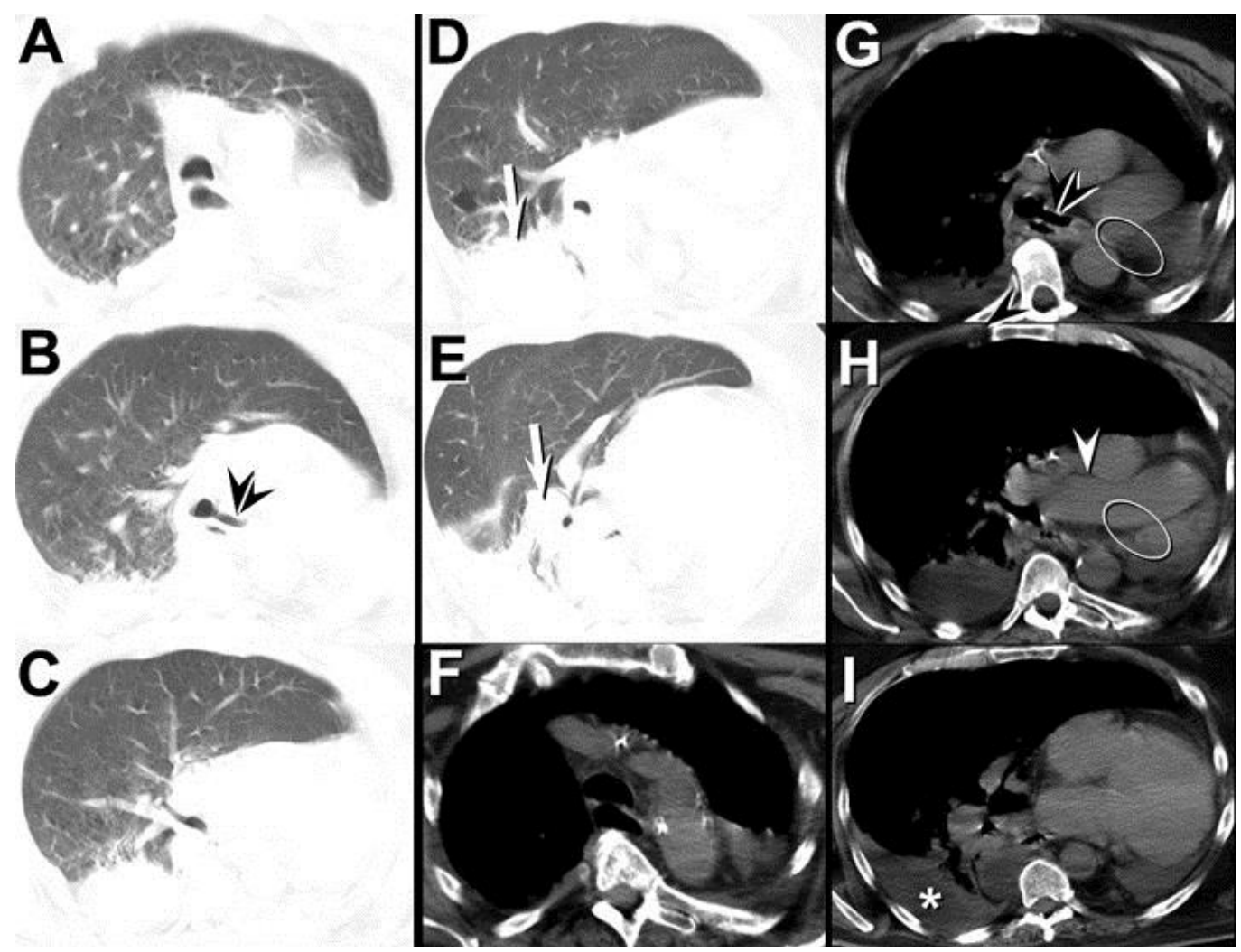

Figure 2. A-F: Representative images form axial unenhanced thoracic CT displayed in lung $(A-E)$ and soft tissue $(F-I)$ windows. 

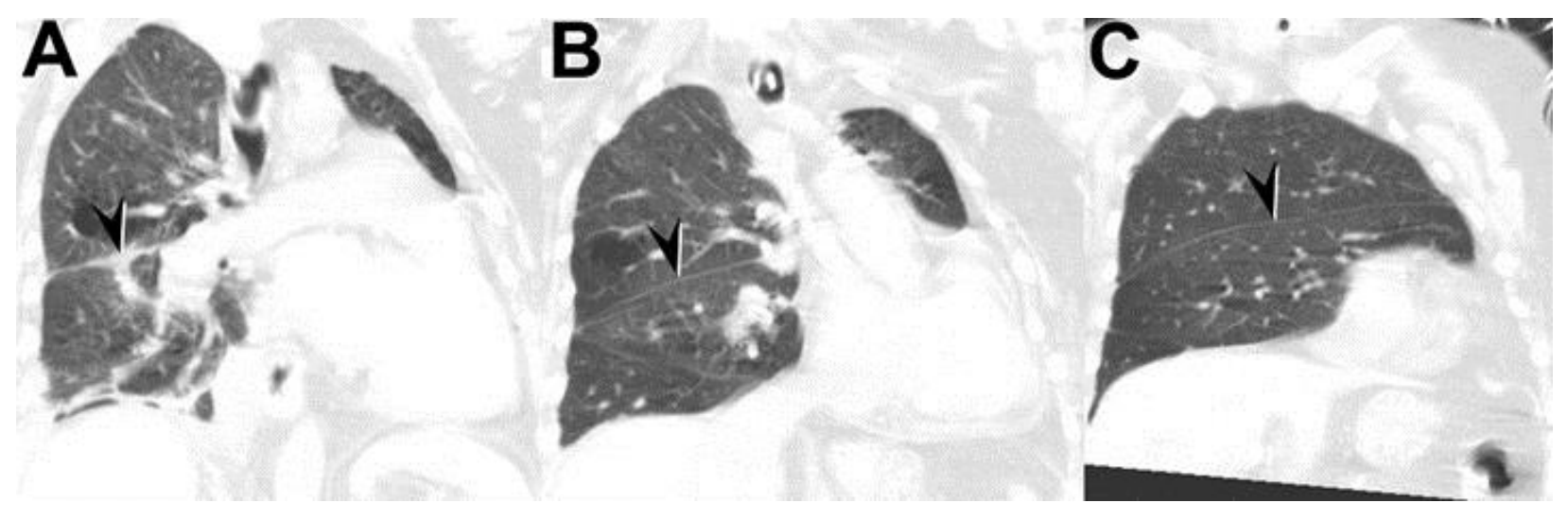

Figure 3. Representative images from coronal unenhanced thoracic CT displayed in lung windows.

Which of the following statements regarding this imaging study is most accurate?

1. The unenhanced thoracic CT shows a large right pneumothorax

2. The unenhanced thoracic CT shows a lesion obstructing the left mainstem bronchus

3. The unenhanced thoracic CT shows a right mainstem bronchial obstruction producing extensive right lung air trapping

4. The unenhanced thoracic CT shows absence of identifiable left lung tissue

5. The unenhanced thoracic CT shows extensive left-sided pleural thickening causing visceral pleural restriction 


\section{Correct!}

\section{The unenhanced thoracic $C T$ shows absence of identifiable left lung tissue}

The right main bronchus and right-sided bronchi appear widely patent- no right-sided endobronchial lesion is seen, and no right lung mosaic perfusion to suggest air trapping is noted. Right lower lobe consolidation and a small right-sided parapneumonic effusion is present. No pneumothorax is noted. A trace amount of left pleural liquid is difficult to exclude, but no significant left-sided pleural thickening is present, and therefore no evidence of left-sided visceral pleural restriction is seen. A rudimentary left mainstem bronchus is present, but appears to end blindly, and no left lung parenchyma is identified. A normal right pulmonary artery is present, but no left pulmonary artery is seen and no left-sided pulmonary venous structures are present. The right lung volume is large, representing compensatory overinflation, and extends across midline to contact the left chest wall. The heart is completely shifted into the left thorax and rotated posteriorly.

Which of the following represents the next most appropriate step for the evaluation of this patient?

1. Continued therapy for the presenting right lower lobe pneumonia

2. Flexible fiberoptic bronchoscopy

3. Mediastinoscopy

4. Thoracentesis

5. Thoracic MR 


\section{Correct! \\ 1. Continued therapy for the presenting right lower lobe pneumonia}

The thoracic CT has excluded significant, reversible derangements causing the extensive left-sided volume loss, and therefore, additional procedures are not required. No active endobronchial obstruction is seen, nor is pleural thickening requiring cortication is noted. Thoracic MR would not add any additional information to what has already been learned with thoracic CT. Unless information suggesting that the right lower lobe bronchopneumonia and presumed right-sided parapneumonic effusion has become complicated or is not responding to therapy appropriately, right-sided thoracentesis is not required; there is no significant left pleural fluid to sample. Conservative management is all that is required.

Which of the following represents the correct etiology for the extensive left-sided volume loss in this patient?

1. Interruption of the left pulmonary artery

2. Left lung agenesis

3. Left lung aplasia

4. Left lung hypoplasia

5. None of the above 


\section{Correct!}

\section{Left lung aplasia}

Pulmonary underdevelopment (Figure 4) has been classified into three broad categories: pulmonary agenesis (Figure 4A), pulmonary aplasia (Figure 4B and C), and pulmonary hypoplasia (Figure 4F and $\mathrm{G}$ ).

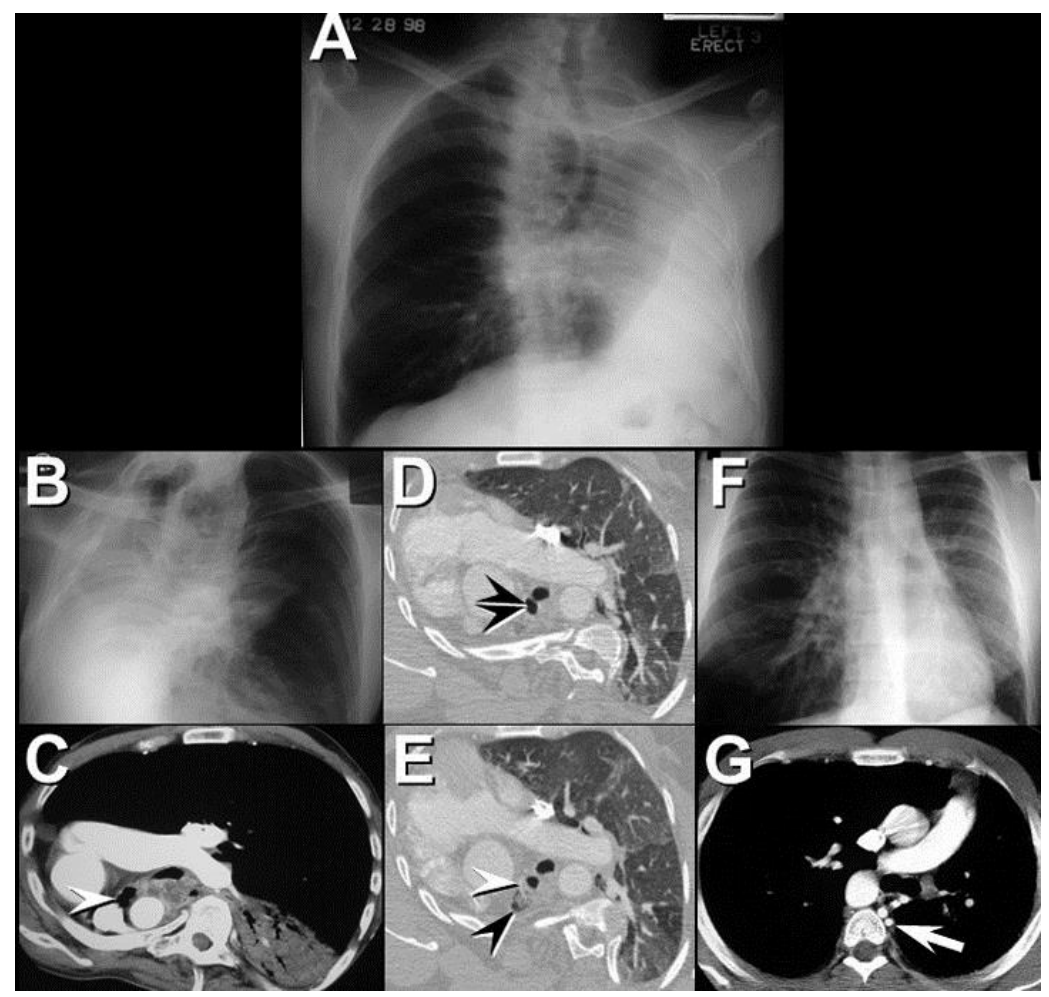

Figure 4. Pulmonary underdevelopment. A: left lung agenesis. Note extensive left lung volume loss with complete shift of cardiomediastinal structures into the left thorax. At CT (not shown), no left pulmonary artery or veins, left mainstem bronchus, or lung tissue was present. B and C: right lung aplasia. Note extensive right lung volume loss at chest radiography. Consolidation in the left base represented pneumonia, which brought the patient to clinical attention. Right lung tissue and a right pulmonary artery are absent, but note the presence of a right mainstem bronchus (white arrowhead, C), which distinguishes pulmonary agenesis from pulmonary aplasia. $\mathrm{D}$ and $\mathrm{E}$ : Right pulmonary hypoplasia. Note presence of a right mainstem bronchus (black double arrowhead, D) and a small amount of rudimentary pulmonary tissue (black arrowhead, E) and a

severely hypoplastic right pulmonary artery (white arrowhead, E) supplying the rudimentary lung tissue. F and G: Interruption of the left pulmonary artery. Note volume loss on the affected left side with absence of the proximal left pulmonary artery.

Although the left thorax is hypoplastic, left lung is present and left-sided bronchi are present. Note presence of systemic arterial supply derived from the bronchial and intercostal arterial circulations (arrow, G) that eventually reconstitutes more distal left pulmonary arterial branches. The aortic arch is right-sided and often interruption of the pulmonary artery occurs on the side opposite of the aortic arch. 
Pulmonary agenesis (Figure 4A) represents complete absence of the lung parenchyma, bronchus, and pulmonary vasculature, whereas the term pulmonary aplasia (Figure 4B and $\mathrm{C}$ ) refers to complete absence of the lung parenchyma and pulmonary vasculature, but with the presence of a blind-ending, rudimentary bronchus, which fits the CT appearance of this patient. Pulmonary hypoplasia (Figure 4D and $\mathrm{E}$ ) is present when a bronchus and rudimentary lung are seen and pulmonary arteries are also present, but are decreased in size and number. Interruption of the pulmonary artery (Figure 4F and $\mathrm{G}$ ) is characterized by volume loss and abrupt truncation of the proximal pulmonary artery on the affected side, with the more distal pulmonary arteries on the affected side reconstituted by systemic vessels, often derived from the bronchial and intercostal circulations. Venous drainage on the affected side is typically normal, and lung, albeit hypoplastic, is present.

Diagnosis: Left lung aplasia presenting due to incidental development of right lower lobe bronchopneumonia

\section{References}

1. Biyyam DR, Chapman T, Ferguson MR, Deutsch G, Dighe MK. Congenital lung abnormalities: embryologic features, prenatal diagnosis, and postnatal radiologicpathologic correlation. Radiographics. 2010 Oct;30(6):1721-38. [CrossRef] [PubMed]

2. Garcia-Pe-a P, Coma A, Enríquez G. Congenital lung malformations: radiological findings and clues for differential diagnosis. Acta Radiol. 2013 Nov;54(9):1086-95. [CrossRef] [PubMed]

3. Kwon $\mathrm{SH}$, Oh JH, Sung DW. Incidentally found right pulmonary aplasia in an adult patient: the 64-slice MDCT findings. J Thorac Imaging. 2009 Feb;24(1):56-8. [CrossRef] [PubMed]

4. Thacker PG, Schooler GR, Caplan MJ, Lee EY. Developmental lung malformations in children: recent advances in imaging techniques, classification system, and imaging findings. J Thorac Imaging. 2015 Jan;30(1):29-43. [CrossRef] [PubMed]

5. Zylak CJ, Eyler WR, Spizarny DL, Stone CH.Developmental lung anomalies in the adult: radiologic-pathologic correlation. Radiographics. 2002 Oct;22 Spec No:S2543. [CrossRef] [PubMed] 